\title{
La solitude:
}

\section{un serial killer à démasquer}

\section{Anne-Françoise Allaz}

Prof. Dr méd., membre de la rédaction

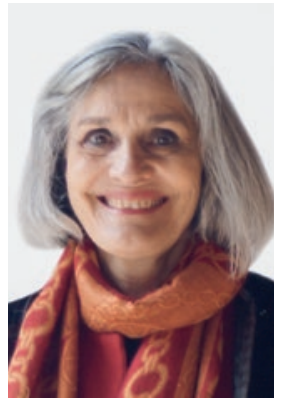

"Le secret d'une bonne vieillesse n'est rien d'autre que la conclusion d'un pacte honorable avec la solitude." G. García Márquez, Cent ans de solitude

Un sondage de la SSR, publié le 6 novembre 2020, révèle que la peur de l'isolement social et de la solitude en lien avec la pandémie est passée en Suisse de 30 à $46 \%$ entre juin et octobre 2020.

Ce sondage fait écho au récent rapport de la Fondation de France sur 10 ans de «solitudes» [1]. On y apprend que l'isolement social augmente et concerne $15 \%$ des résidents français. Ces isolés sont «défiants» ou «en souffrance» (38\%), aussi bien que "sereins» ou "seuls par choix» (43\%). L'isolement social touche principalement les personnes en situation précaire ou âgées, mais aussi, et de manière préoccupante, de plus en plus les jeunes. En Suisse, l'Office fédéral de la statistique (OFS) indiquait en 2017 que 38\% des résidents déclaraient souffrir de solitude ainsi que $46 \%$ des migrants de première génération [2].

Références

1 Berhuet S, Mansencal LB, Etienne L, et al. Dix ans d'observation de l'isolement relationnel: un phénomène en forte progression. Les solitudes en France Fondation de France. 2020.

2 Enquête suisse sur la santé. OFS, 2019.

3 Holt-Lunstad J, et al. Social Relationships and Mortality Risk: A Meta-analytic Review. PLoS Med. 2010;7(7):e1000316.

4 Bzdok D, Dunbar R. Neurobiology of Social distancing. Trends in Cognitive Sciences. 2020;24. 717-33.

afallaz[at]bluewin.ch
Isolement social et solitude sont liés sans être équivalents. Le premier est mesurable par la fréquence des contacts, l'intégration dans un réseau. Quant au sentiment de solitude, "il est l'expression subjective d'un manque de ressources sociales ou d'un besoin de contacts sociaux supplémentaires ou différents", selon l'OFS.

En cette période de Covid, avec son cortège de deuils, de confinement et de précarisation, l'impact de la perte des liens sociaux sur la santé prend un relief particulier. Au fond, qu'en savons-nous?

Pour résumer, il existe depuis des années des évidences solides que le manque d'intégration sociale augmente significativement le risque de mortalité précoce, notamment cardiovasculaire. "La magnitude de ce risque dépasse celle de plusieurs facteurs de risque classiques», écrit l'auteure d'une méta-analyse qui inclut plus de 300000 patients [3]. «La solitude plus mortelle que l'obésité», résumait Bertrand Kiefer dans la Revue Médicale Suisse.

Une étude récente complète ce sombre tableau avec des données sur les atteintes immunitaires et le risque de maladie d'Alzheimer liés à la privation sociale ou à la solitude [4]. Cela s'ajoute aux conséquences psychologiques mieux reconnues, comme les dépressions et les suicides, ou aux répercussions sur la qualité de vie, l'image de soi ou encore le renoncement aux soins.

A l'aune de ces données, l'on peut s'étonner du relatif silence qui entoure ce déterminant pourtant majeur de la santé. Que l'homme de tout âge soit un être éminemment social, nous le savions. Nous avons le goût des autres. Nous avons besoin - un besoin vital d'interactions, de paroles, d'émotions, de contacts physiques. Leur manque nous rend vulnérables aux maladies et peut jusqu'à nous tuer. Souvenez-vous de l'effrayante surmortalité des enfants élevés sans attention ou affection dans des orphelinats - sans même mentionner leurs graves troubles de développement.

Or, l'isolement social est en forte progression partout en Occident. L'OMS s'en préoccupe. Les gouvernements également, qui créent des ministères, comme celui de la Loneliness en Angleterre (sic!), et maintes commissions pour la combattre. Les municipalités et de nombreuses associations admirables s'engagent pour favoriser le lien social et la solidarité. Heureusement, car la souffrance doit être très profonde de n'avoir personne à qui se confier.

Et nous les médecins? Nous sommes avec les pharmaciens et le personnel soignant à domicile en première ligne des rencontres avec les personnes isolées. Ce sujet dérangeant ne devrait pas être évité pour des raisons autant médicales qu'humanistes. Si nous prenons conscience de cet enjeu, nous pouvons agir à divers niveaux. Que ce soit en influençant la politique de la santé ou en soutenant les institutions qui créent du lien et contribuent à la réinsertion sociale. Mais aussi en mettant son patient en contact direct avec un réseau, une association ou des ressources disponibles en cas de détresse. Tous ces actes d'apparence banale peuvent améliorer la santé et - pourquoi pas - influencer une trajectoire. Une main tendue peut changer une vie, comme nous l'avons appris des spécialistes de la résilience. Aussi, est-ce une année 2021 très solidaire que je vous souhaite! 